\title{
Differential expression of ETS family members in prostate cancer tissues and androgen-sensitive and insensitive prostate cancer cell lines
}

\author{
미미 \$ 9,' [\$ ' / ( $5 *$, ANDREAS LINDSTROT*, BERIT LANGER, \\ REINHARD BUETTNER and NICOLAS WERNERT \\ Institute of Pathology, University Hospital Bonn, D-53127 Bonn, Germany
}

Received November 17, 2010; Accepted January 19, 2011

DOI: $10.3892 /$ ijmm.2011.672

\begin{abstract}
The ETS family of transcription factors plays important roles in both normal and neoplastic cells for different biological processes such as proliferation, differentiation, development, transformation, apoptosis, migration, invasion and angiogenesis. The 27 ETS factors are probably a part of complex regulatory networks including interactions among family members. In human prostate cancer, rearrangements have been found in several genes of the ETS family resulting in chimeric oncoproteins. In a previous study we found that the ETS family prototype, Ets-1 affects biological properties of PC3 prostate cancer cells. In a first effort to understand the cooperative interactions between different ETS factors in prostate cancer, in the present study we examined the expression pattern of all 27 ETS members using quantitative RT-PCR (qRT-PCR) in the androgen-sensitive VCaP and LNCaP, and the androgen-insensitive PC3 and DU-145 prostate cancer cell lines as well as in human prostate cancer tissue samples. We further investigated whether the ETS family prototype, Ets-1, regulates other ETS family members by examining the effect of Ets-1 blockade in PC3 cells on their expression. We found an expression specificity of various ETS family members in the prostate cancer cell lines which might reflect their different biological properties. In human prostate samples only 3 among the 27 ETS family members (Ehf, Elk-4 and Ets-2) showed significant expression differences between normal and cancerous prostate glands. We finally demonstrate that the family prototype, Ets-1, regulates the family members Elf-1, Elf-2, Elk-1, Etv-5 and Spi-1 in PC3 prostate cancer
\end{abstract}

Correspondence to: Professor Nicolas Wernert, Institute of Pathology, University Hospital Bonn, Sigmund-Freud Str. 25, D-53127 Bonn, Germany

E-mail: nicolas.wernert@ukb.uni-bonn.de

*Contributed equally

Key words: transcriptional regulation, Ets-1, LNCaP, VCaP, PC3, DU-145 cells. Chimeric oncoproteins containing ETS family members arising due to frequent translocations in prostate cancer are probably part of a regulatory network involving other ETS family members as well.

\section{Introduction}

The ETS family of transcription factors consists of 27 members in humans (1) and distinct ETS family members can play important roles for quite different processes such as development, differentiation, proliferation, apoptosis, migration, tissue remodelling, invasion and angiogenesis in various cell types including B cells, endothelial cells, fibroblasts as well as diverse neoplastic cells (2-6).

In human malignant tumours, several genes of the ETS family are rearranged to produce chimeric oncoproteins (7). In human prostate cancers (PCA) translocations between the TMPRSS2 gene, an androgen-regulated prostate-specific serine protease, with several members of the ETS family such as Etv-1, Etv-4, Etv-5 and most commonly Erg, are frequently reported (6,8-10). The consequences of these translocations in PCA result in increased expression of the rearranged ETS factors as a response to androgens (6). Studies have suggested that the ETS rearrangements are sufficient for initiation of prostate neoplasia, while most recently other investigations have suggested that the rearrangements may foster progression rather than initiation events in prostate tumourigenesis $(11,12)$.

All ETS transcription factors are characterized by an evolutionarily highly conserved DNA-binding domain, the ETS domain (1). Based on phylogenetic analysis of this ETS domain, subfamilies of more highly related members were identified although the DNA-binding properties of ETS proteins were found to be similar due to the amino acid conservation within the ETS domains (13). Nonetheless, binding preferences for distinct flanking sequences could facilitate specificity of ETS-factor binding and help to explain unique biological functions of different ETS proteins (13). Furthermore, redundant occupancy of ETS family members at proximal regulatory gene regions and overlapping functions for ETS factors have been reported $(13,14)$. It is also conceivable, that different ETS members can bind to the same 
ETS-binding sites (EBS) in the regulatory regions of various target genes with different affinities and thereby compete for binding. The specificity and degree of trans-activation (or trans-repression) for a given target gene could then result from the dynamic binding equilibrium, the activity of ETS-transactivation domains, as well as from ternary complex formation of different ETS members with other transcription factors (15). Complexity of such regulations concerning in particular different ETS family members could also play a role in the development and progression of PCA with or without translocations of the TMPRSS2 gene. Among ETS family members, Ets-1 is the prototype, which is expressed in various cell types and found to be implicated in tumour angiogenesis and invasion (16-18). Previously, we reported that Ets-1 promotes proliferation, migration or invasion in various neoplastic cells including melanoma, HeLa, glioma as well as PC3 prostate cancer cells $(4,5,19,20)$. In a first effort to understand the cooperative interactions of different ETS factors in PCA, we examined the expression pattern of all 27 members of the ETS family in a selected group of androgen-sensitive (LNCaP and $\mathrm{VCaP}$ ) and -insensitive (PC3 and DU-145) prostate cancer cell lines as well as in human prostate cancer tissues. We also investigated whether the ETS family prototype, Ets-1, regulates other ETS family members in PC3 cells.

We found a unique differential expression pattern of the 27 ETS family members among the prostate cancer cell lines and demonstrated that the family prototype, Ets-1, actually regulates the ETS factors Elf-1, Elf-2, Elk-1, Etv-5 and Spi-1 in PC3 prostate cancer cells. Only 3 among the 27 ETS members (Ehf, Elk-4 and Ets-2) showed a significant difference in expression between human samples of normal prostate glands and tumour glands.

Chimeric oncoproteins containing ETS family members in prostate cancer are therefore probably part of a regulatory network involving other ETS family members as well.

\section{Materials and methods}

Cell culture. LNCaP, VCaP, PC3 and DU-145 cells were grown in RPMI (Invitrogen, USA), DMEM (ATCC, USA), F12K and MEM (Invitrogen) media, respectively. The media were supplemented with $0.5 \%$ penicillin/streptomycin (Invitrogen) and $10 \%$ heat-inactivated fetal calf serum (Invitrogen). The MEM and RPMI media were additionally supplemented with $1 \%$ glucose, $1 \%$ sodium pyruvate and $1 \%$ non-essential amino acids (NEIAA) (all from Invitrogen). The media of transfected PC3 cells were additionally supplemented with $4.3 \mathrm{mg} / \mathrm{ml}$ of G-418 (PAA, Austria).

Ets-1 block. PC3 cells were cultured in F12K media supplemented with antibiotics and $10 \%$ heat-inactivated fetal calf serum. Plasmids pcDNA3.1h-ets-1 inverse and pcDNA3.1(-) (mock control), respectively, were transfected into the cells by the calcium phosphate method as previously described (21). At $24 \mathrm{~h}$ after transfection, selection was started using $4.3 \mathrm{mg} / \mathrm{ml} \mathrm{G}-418$ (PAA). The cells were then lysed by freeze and thaw at room temperature. A total of $20 \mu \mathrm{g}$ of protein was analyzed by $10 \%$ SDS-polyacrylamide gel electrophoresis and transferred onto nitrocellulose (Bio-Rad, USA). The filters were then blocked with 5\% dry milk in TBST (50 mM Tris, $150 \mathrm{mM}$
$\mathrm{NaCl}, 0.1 \%$ Tween-20, $\mathrm{pH}$ 7.5) for $2 \mathrm{~h}$ at room temperature followed by incubation in TBST with an anti-Ets-1 mouse monoclonal antibody (Transduction Laboratories, USA), then with an anti-mouse IgG horseradish peroxidase-conjugated secondary antibody (Amersham Biosciences, England). Proteins were visualized using ECL reagents (Amersham Biosciences). The housekeeping protein, $\beta$-actin, was used as an internal control and was detected using mouse monoclonal anti- $\beta$-actin antibody (Sigma, Germany).

Quantitative RT-PCR. RNA was extracted using Qiagen's RNeasy mini kit (Germany) as described by the manufacturer. Reverse transcription was performed on the RNA using SuperScript III First-Strand Synthesis SuperMix for qRT-PCR (Invitrogen) as described by the manufacturer. For the real-time PCR a total volume of $10 \mu 1$ SYBR-GreenER qPCR SuperMix (Invitrogen) was used. The oligonucleotides and sequences for the 27 ETS family members were designed as previously described (6). The housekeeping gene oligonucleotides were as follows, rPL13a (forward) TACGCTGTGAAGGCATCAAC, rPL13a (reverse) CACCATCCGCTTTTTCTTGT. Samples were analyzed in triplicate. The PCR conditions in the Applied Biosystems $7900 \mathrm{HT}$ instrument were $2 \mathrm{~min}$ at $50^{\circ} \mathrm{C}, 10 \mathrm{~min}$ at $95^{\circ} \mathrm{C}$ and 40 cycles of $15 \mathrm{sec}$ at $95^{\circ} \mathrm{C}$ and for $1 \mathrm{~min}$ at $60^{\circ} \mathrm{C}$. After 40 rounds of cycling, a melting point analysis was performed with $15 \sec 95^{\circ} \mathrm{C}, 15 \sec 60^{\circ} \mathrm{C}$ and $15 \sec 95^{\circ} \mathrm{C}$. The data were collected after the $60^{\circ} \mathrm{C}$ step at every cycle and after the melting point analysis.

Data processing. Absolute Ct-values were assessed with the SDS 2.2 software (Applied Biosystems, USA) and transferred to Microsoft Excel for further analysis. The Ct-values were normalized with the reference rPL13a as the housekeeping gene. After that, the $2^{-\Delta \Delta C t}$ values were calculated with the values of the mock control in the cell culture used as a second reference. SEM is shown in the error bar. Significance was calculated with the Student's t-test included in MS Excel. $\mathrm{P}$-values are indicated as ${ }^{* * *} \mathrm{P}<0.001,{ }^{* *} \mathrm{P}<0.01$ and ${ }^{*} \mathrm{P}<0.05$.

Processing of human prostatectomy specimens. Fresh tissue samples from 5 patients with moderately differentiated prostate carcinomas (Gleason scores 6 and 7) were taken immediately after radical prostatectomy and shock-frozen in liquid nitrogen with ice-cold isopentane. Samples were taken from both, the normal prostate proper and from carcinomas. Sample sizes were around $0.5 \times 0.5 \times 0.3 \mathrm{~cm}$. The patients' age ranged from 63-72 years (average of 66.6 years). Frozen sections of $6 \mu \mathrm{m}$ thick were cut from the samples using a cryotome (Leica, Germany) and mounted on membrane-coated slides (1 $\mathrm{mm}$ PEN, Zeiss, Germany) for subsequent laser-microdissection. One section was mounted on conventional slides and stained with haematoxylin and eosin (H\&E) for diagnostic evaluation by an experienced pathologist. Laser-microdissection was carried out according to our previously published methods (22-24). Frozen sections were dried for $2 \mathrm{~min}$ in the cryotome, washed for 2 min with $70 \%$ ethanol in DEPC-treated water and stained for $30 \mathrm{sec}$ in $1 \%$ Cresyl Violet diluted in $50 \%$ ethanol-DEPC-treated water. Slides were then washed briefly in 70 and $100 \%$ ethanol, dried for $10 \mathrm{~min}$ and stored at $-80^{\circ} \mathrm{C}$ until use for laser-microdissection. 

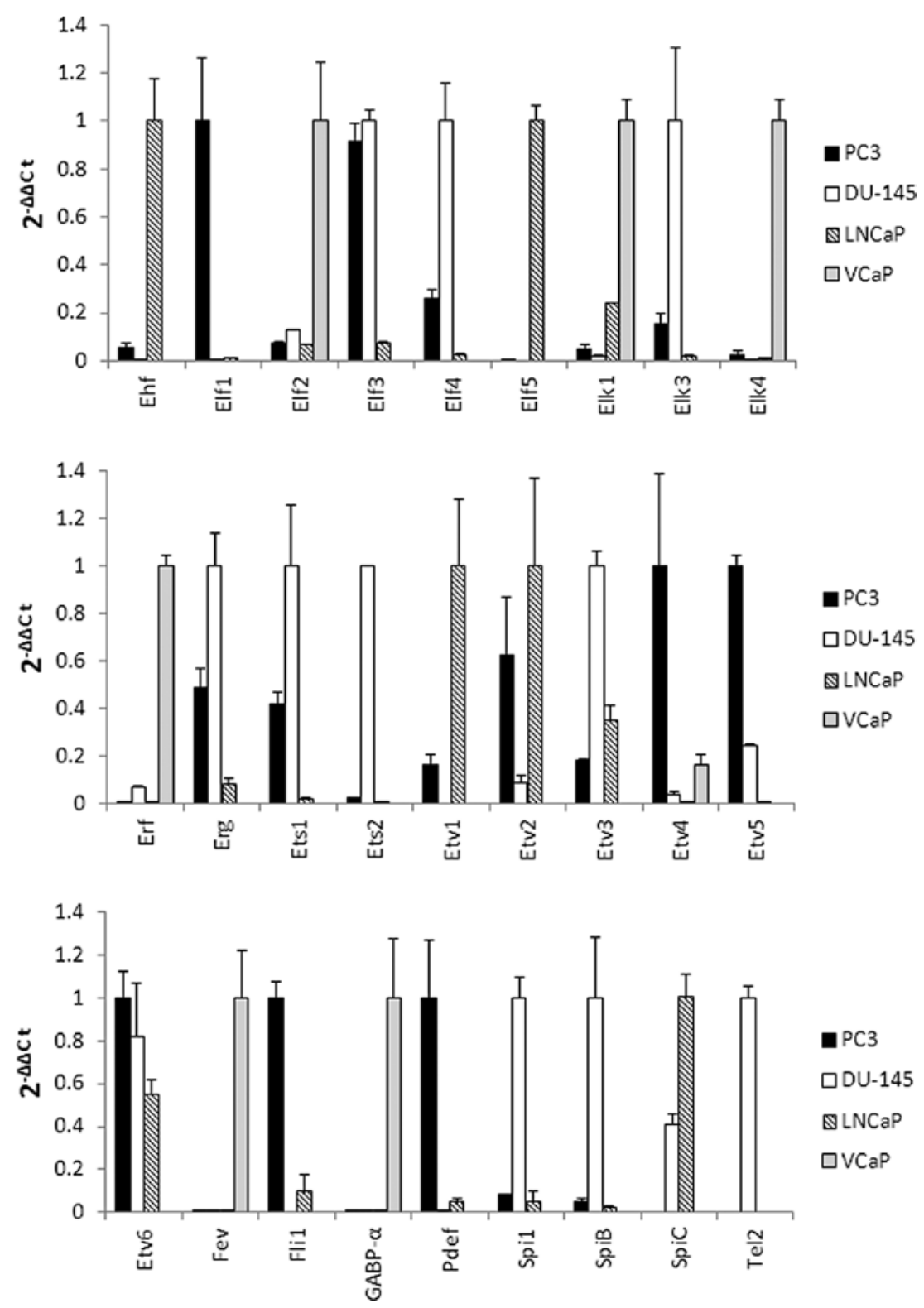

Figure 1. Expression profiles of the ETS family in prostate cancer cell lines. The expression of the 27 ETS family members was measured by quantitative RT-PCR in LNCaP, VCaP, PC3 and DU-145 prostate cancer cell lines.

Quality control. One frozen section from each sample was used for control of RNA integrity prior to laser-capture microdissection (LCM) using the laser capture microscope (Axio Observer Z1, Zeiss). The section was washed from the slide with $600 \mu 12 \mu \mathrm{M}$ DTT in RLT buffer (provided by the RNeasy mini kit, Qiagen) and vortexed for 30 sec. RNA extraction was performed as described by the manufacturer. The recommended DNase digestion was carried out with an RNase-free DNase set (Qiagen). RNA integrity was measured with the Agilent Bioanalyzer 2100 (Agilent Technologies, USA). Samples with a RIN factor $>6$ were used for LCM.

Laser-capture microdissection. LCM of Cresyl Violetstained sections was performed using the Axio Observer Z1 microscope with the integrated PALM MicroBeam software (Zeiss). LCM was carried out under a 10X objective. From every patient normal and cancerous glands were cut out. Isolated normal or cancerous glands were collected in $200 \mu 1$ adhesive cap tubes (Zeiss). Total RNA was isolated from samples with the RNeasy Micro kit (Qiagen) as described by the manufacturer. The recommended DNase digestion was carried out with the RNase-free DNase set. The amount of the isolated RNA was determined using an ND1000 Nanodrop (Thermo Fisher Scientific, USA).

\section{Results}

Expression profiles of the ETS family in prostate cancer cell lines. Using quantitative RT-PCR, we determined the expression profile of the 27 ETS family members in LNCaP, VCaP, PC3 and DU-145 prostate cancer cell lines (Fig. 1). The figure shows that each of the cell lines has a unique expression profile of the different ETS family members.

Expression profile of the ETS family members in prostate cancer tissues. Using quantitative RT-PCR analysis, the 


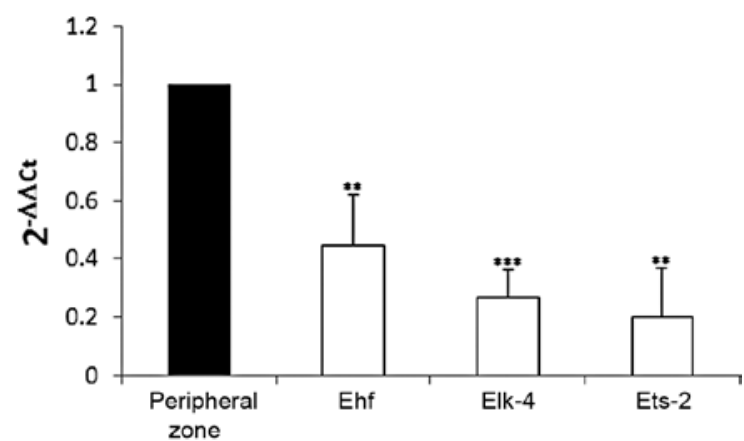

Figure 2. The expression of the 27 ETS-family members in prostate cancer tissues. The expression of the ETS family members were measured in the tissues with quantitative RT-PCR and were compared between microdissected healthy prostate glands and tumour glands (Gleason scores 6 and 7) from 5 patients. A significant down-regulation in the tumourous glands compared to the normal glands was observed for Ehf, Elk-4 and Ets-2. Significance was calculated with the Student's t-test. ${ }^{* * *} \mathrm{P}<0.001,{ }^{* *} \mathrm{P}<0.01$.

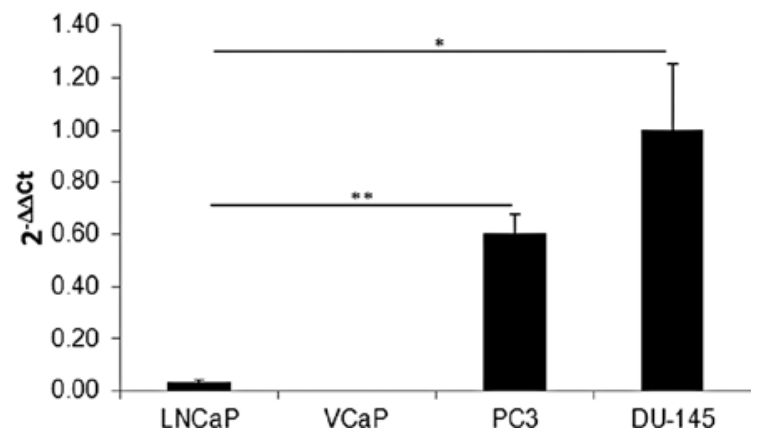

Figure 3. Expression of Ets-1 in prostate cancer cell lines. The relative expression of Ets-1 as measured by quantitative RT-PCR was compared among the DU-145, PC3, VCaP and LNCaP prostate cancer cell lines. Significance was calculated with the Student's t-test. ${ }^{* *} \mathrm{P}<0.01$ and ${ }^{*} \mathrm{P}<0.05$.

expression of the ETS family members was measured in normal prostate glands and tumourous glands from moderately differentiated prostate carcinomas which had been isolated by laser microdissection (Fig. 2). As demonstrated in the figure the ETS factors Ehf, Elk-4 and Ets-2 show significant down-regulation in the tumourous glands compared to the healthy glands.

Expression of Ets-1 in prostate cancer cell lines. Based on quantitative RT-PCR analysis, we compared the expression level of the ETS family prototype, Ets-1, among LNCaP, VCaP, PC3 and DU-145 prostate cancer cell lines (Fig. 3). As shown in the figure that Ets-1 is highly expressed in the androgen-insensitive DU-145 and PC3 cell lines compared to the androgen-sensitive $\mathrm{LNCaP}$ and $\mathrm{VCaP}$ cells.

Experimental blocking of Ets-1 in PC3 prostate cancer cells. Two stable PC3 cell cultures were established by transfection with either an Ets-1 inverse antisense expression vector or a mock control vector. Using Western blot analysis, we found that PC3 cells expressing the mock control showed detectable amounts of Ets-1, whereas PC3 cells expressing the Ets-1 inverse lacked detectable amounts of Ets-1 over a period of 121 days (20).

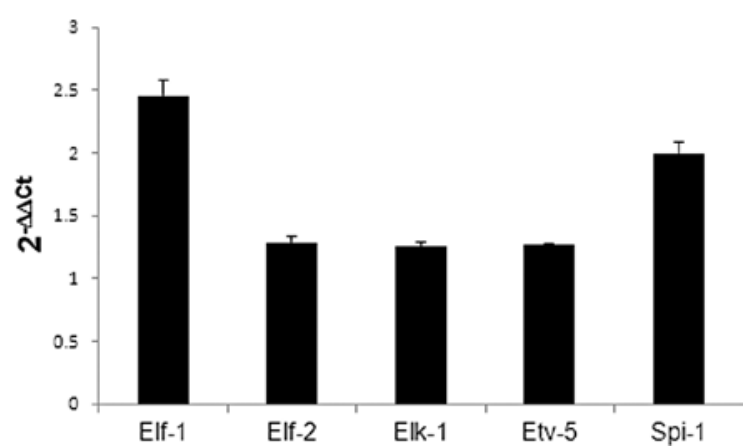

Figure 4. Ets-1 regulates other ETS family members in PC3 prostate cancer cell lines. Quantitative RT-PCR analysis using RNA extracted from Ets-1 blocked and mock control PC3 cells shows an up-regulation of the ETS family members Elf-1, Elf-2, Elk-1, Etv-5 and Spi-1 in the Ets-1 blocked cells compared to the mock control.

Ets-1 regulates other ETS family members in PC3 prostate cancer cell lines. Quantitative RT-PCR analysis using RNA extracted from Ets-1 blocked and mock control PC3 cells demonstrated an up-regulation of the ETS family members Elf-1, Elf-2, Elk-1, Etv-5 and Spi-1 in the Ets-1 blocked cells compared to the mock control (Fig. 4).

\section{Discussion}

The ETS family of transcription factors is involved in many biological processes in both normal and neoplastic cells (2-6). There are 27 known ETS family members in humans and all are characterized by an evolutionarily highly conserved DNA-binding domain, the ETS domain (1). Although, the DNA-binding properties of ETS proteins are similar, different ETS proteins may exhibit preference for distinct flanking sequences in regulatory gene regions that could facilitate specificity and explain their unique biological functions (13). Redundant occupancy of ETS family members at proximal regulatory gene regions as well as overlapping functions have already been reported $(13,14)$. The specificity and degree of transactivation for given target genes could result from dynamic binding equilibrium as well as from ternary complex formation of different ETS members with other transcription factors (15). Complex regulations involving different ETS family members could also concern prostate cancers with or without translocations of the TMPRSS2 gene $(6,8-10)$ and play a role in tumour development and progression.

In a first attempt to understand this possible network, we analyzed the mRNA expression level of all the ETS family members in the androgen-sensitive, $\mathrm{LNCaP}$ and $\mathrm{VCaP}$, and the androgen-insensitive, PC3 and DU-145, prostate cancer cell lines. Our data show, that each of the cell lines has a unique expression profile of the ETS family members. However, due to similar DNA binding specificities of ETS factors, it is not possible to determine which family member may regulate the expression of others. Therefore, we decided to first examine whether the ETS family prototype, Ets-1, regulates other family members. We found Ets-1 to be highly expressed in the androgen-insensitive DU-145 and PC3 compared to the androgen-sensitive $\mathrm{LNCaP}$ and $\mathrm{VCaP}$ cell lines. Since we had found in a previous study that Ets-1 has an effect upon biological properties of PC 3 cells (20), we chose this cell 
line to perform further experiments by blocking Ets-1. We actually observed an up-regulation of the family members Elf-1, Elf-2, Elk-1, Etv-5 and Spi-1 in the Ets-1 blocked cells compared to the mock control and thereby present the first evidence that ETS family members regulate each other in PCA. Interestingly, expression of Ets-1 has already been shown in human prostate cancer tissues (16), where it might also regulate other ETS family members in tumours with or without chimeric TMPRSS2-ETS fusions.

The unique expression patern of the ETS family members among the four cell lines might reflect different biological properties of the cells. The four cell lines are derived from different origins, where DU-145, PC3, LNCaP and VCaP cells are respectively derived from a brain metastasis, an advanced androgen-independent bone metastasis, a supraclavicular lymph node metastasis and a metastatic lesion to a lumbar vertebral body of a patient with hormone refractory prostate cancer. Furthermore, the $\mathrm{LNCaP}$ and $\mathrm{VCaP}$ cell lines can serve as a model for the rearrangement of ETS factors due to the presence of translocations in Etv-1 and Erg, respectively. Different expression patterns of the 27 ETS members could reflect their mechanistic complexity and stress that competition among family members based on their abundance, affinity and other co-factors may regulate their functions.

In human prostate samples we found that only 3 among the 27 ETS members, Ehf, Elk-4 and Ets-2, show a significant difference in expression between normal prostate glands and prostate cancer glands. These three family members were found to be down-regulated in the tumour glands compared to the normal glands. All 3 ETS-factors are also differentially regulated in the 4 prostate cancer cell lines with the highest values found for Ehf in $\mathrm{LNCaP}$, for Elk-4 in $\mathrm{VCaP}$ and for Ets-2 in DU-145 cells. It will be worth targeting these ETS-factors in the different cell lines in order to characterize their possible functions.

\section{Acknowledgements}

We are grateful to the DFG(Deutsche Forschungsgemeinschaft; German Research Association, grant no. WE 1104/11-1) and the German Cancer Aid (Deutsche Krebshilfe, grant no. 107827) for financial support.

\section{References}

1. Hollenhorst PC, Jones DA and Graves BJ: Expression profiles frame the promoter specificity dilemma of the ETS family of transcription factors. Nucleic Acids Res 32: 5693-5702, 2004.

2. Oikawa T: ETS transcription factors: possible targets for cancer therapy. Cancer Sci 95: 626-633, 2004.

3. Hashiya N, Jo N, Aoki M, et al: In vivo evidence of angiogenesis induced by transcription factor Ets-1: Ets-1 is located upstream of angiogenesis cascade. Circulation 109: 3035-3041, 2004.

4. Rothhammer T, Hahne JC, Florin A, et al: The Ets-1 transcription factor is involved in the development and invasion of malignant melanoma. Cell Mol Life Sci 61: 118-128, 2004.
5. Hahne JC, Okuducu AF, Kaminski A, Florin A, Soncin F and Wernert N: Ets-1 expression promotes epithelial cell transformation by inducing migration, invasion and anchorage-independent growth. Oncogene 24: 5384-5388, 2005.

6. He J, Pan Y, Hu J, Albarracin C, Wu Y and Dai JL: Profile of Ets gene expression in human breast carcinoma. Cancer Biol Ther 6: 76-82, 2007.

7. Hahne JC, Okuducu AF, Sahin A, Fafeur V, Kiriakidis S and Wernert N: The transcription factor ETS-1: its role in tumour development and strategies for its inhibition. Mini Rev Med Chem 8: 1095-1105, 2008

8. Tomlins SA, Rhodes DR, Perner S, et al: Recurrent fusion of TMPRSS2 and ETS transcription factor genes in prostate cancer. Science 310: 644-648, 2005.

9. Furusato B, Tan SH, Young D, et al: ERG oncoprotein expression in prostate cancer: clonal progression of ERG-positive tumor cells and potential for ERG-based stratification. Prostate Cancer Prostatic Dis 13: 228-237, 2010.

10. Kumar-Sinha C, Tomlins SA and Chinnaiyan AM: Recurrent gene fusions in prostate cancer. Nat Rev Cancer 8: 497-511, 2008.

11. Tomlins SA, Laxman B, Dhanasekaran SM, et al: Distinct classes of chromosomal rearrangements create oncogenic ETS gene fusions in prostate cancer. Nature 448: 595-599, 2007.

12. Carver BS, Tran J, Chen Z, et al: ETS rearrangements and prostate cancer initiation. Nature 457: E1-E3, 2009.

13. Hollenhorst PC, Shah AA, Hopkins C and Graves BJ: Genomewide analyses reveal properties of redundant and specific promoter occupancy within the ETS gene family. Genes Dev 21: 1882-1894, 2007.

14. Wei G, Srinivasan R, Cantemir-Stone CZ, et al: Ets1 and Ets2 are required for endothelial cell survival during embryonic angiogenesis. Blood 114: 1123-1130, 2009.

15. Wei GH, Badis G, Berger MF, et al: Genome-wide analysis of ETS-family DNA-binding in vitro and in vivo. EMBO J 29: 2147-2160, 2010.

16. Alipov G, Nakayama T, Ito M, et al: Overexpression of Ets-1 proto-oncogene in latent and clinical prostatic carcinomas. Histopathology 46: 202-208, 2005

17. Wernert N, Gilles F, Fafeur V, et al: Stromal expression of c-Ets transcription factor correlates with tumor invasion. Cancer Res 54: 5683-5688, 1994.

18. Wernert N, Raes MB, Lassalle P, et al: c-ets1 proto-oncogene is a transcription factor expressed in endothelial cells during tumor vascularization and other forms of angiogenesis in humans. Am J Pathol 140: 119-127, 1992.

19. Sahin A, Vercamer C, Kaminski A, et al: Dominant-negative inhibition of Ets 1 suppresses tumor growth, invasion and migration in rat C6 glioma cells and reveals differentially expressed Ets 1 target genes. Int J Oncol 34: 377-389, 2009.

20. Adler D, Langer B, Lindstrot A, etal: Ets-1 is implicated in the regulation of androgen co-regulator FHL2 and reveals specificity for migration, but not invasion, of PC3 prostate cancer cells. Oncol Rep 25: 1125-1129, 2011.

21. Chen CA and Okayama H: Calcium phosphate-mediated gene transfer: a highly efficient transfection system for stably transforming cells with plasmid DNA. Biotechniques 6: 632-638, 1988.

22. Okuducu AF, Hahne JC, Von Deimling A and Wernert N: Laserassisted microdissection, techniques and applications in pathology (Review). Int J Mol Med 15: 763-769, 2005.

23. Okuducu AF, Janzen V, Hahne JC, Ko Y and Wernert N: Influence of histochemical stains on quantitative gene expression analysis after laser-assisted microdissection. Int J Mol Med 11: 449-453, 2003.

24. Wellmann A, Wollscheid V, Lu H, et al: Analysis of microdissected prostate tissue with ProteinChip arrays - a way to new insights into carcinogenesis and to diagnostic tools. Int $\mathbf{J}$ Mol Med 9: 341-347, 2002. 